\title{
NANOCRYSTALLINE DIAMOND IN Ru-DOPED DLC FILMS
}

\author{
G. D. Lian*, E. C. Dickey*, M. Ueno** and M. K. Sunkara** \\ *Department of Materials Science and Engineering, The Pennsylvania State University, University \\ Park, PA 16802. \\ ** Department of Chemical Engineering, University of Louisville, Louisville, KY 40292
}

The properties of diamond like carbon (DLC) films can be significantly enhanced by the presence of nanocrystalline diamond ${ }^{1)}$. Furthermore, metal doping can make DLC films applicable for the electrochemical electrodes used in highly corrosive, aggressive environments ${ }^{2)}$. Here we report the characterization of diamond nanocrystallites in pure and Ru-doped DLC films

The DLC films were deposited by electron cyclotron resonance (ECR) chemical vapor deposition $(\mathrm{CVD})^{3)}$. For $\mathrm{Ru}$ doping, Bis-(ethylcyclopentadienyl)-ruthenium $\left(\mathrm{Ru}\left(\mathrm{C}_{5} \mathrm{H}_{4} \mathrm{C}_{2} \mathrm{H}_{5}\right)_{2}\right.$ was used as the ruthenium precursor. Transmission electron microscopy (TEM) and electron energy loss spectroscopy (EELS) were performed on a JEOL JEM 2010F field-emission transmission electron microscope, operated at $200 \mathrm{kV}$ and equipped with an annular dark field detector, scanning unit, post column image filter (Gatan GIF200) and Oxford energy dipersive x-ray (EDX) detector.

Figure 1 shows high-resolution images of pure and Ru-doped DLC thin films. Diamond nanocrystallites were observed in both samples. The average sizes of the NCD were $11 \mathrm{~nm}$ and $3 \mathrm{~nm}$ in the pure and Ru-doped samples, respectively. Typical selected area diffraction (SAD) patterns from the two samples are shown in Figure 2. The SAD patterns obtained from the both samples are the same. The observed d-spacings are all very closed to that of cubic diamond with space group Fd3m except that the forbidden diamond reflections (002) and (222) were consistently observed in the SAD. These unusually intense forbidden reflections have been interpreted as an FCC polymorph of diamond called n-diamond ${ }^{4)}$. EDX spectra (not shown) indicate that the $\mathrm{Ru}$ is associated with the NCD phase, perhaps residing at the interface between the matrix and crystalline phase. Figure 3 presents EELS spectra of C K edge taken from areas with and without diamond nanocrystallites, and C60 that is used as a standard for $100 \% \mathrm{sp}^{2}$ bonding. In the matrix region of the Ru-doped DLC film the $\mathrm{sp}^{2}$ bonding is $100 \%$ indicating no diamond-like nature to the amorphous carbon. From spectra collected in the areas containing $\mathrm{NCD}$, the total $\mathrm{sp}^{3}$ was calculated to be $15 \%$. The specimen thickness was calculated to be 37.4nm from EELS low-loss spectrum, which translates into the NCD occupying $14 \%$ of the film thickness using the particle size measured from ADF image (figure $3 b$ ).

\section{References}

1. H. Yoshikawa, C. Morel, and Y. Koga, Diamond Related Materials, 10, 1588 (2001).

2. P. Koduri, M. K. Sunkara, E. C. Dickey, C. Frazier, and S. V. Babu Surface Eng. 15, 373 (1999).

3. G. D. Lian, E. C. Dickey, M. Ueno and M.K. Sunkara to be submitted.

4. H. Hirai and K. Kondo, Science 253, 722 (1991).

5. This work was supported by the NSF-MRSEC for Advanced Carbon Materials (DMR-9809686). 

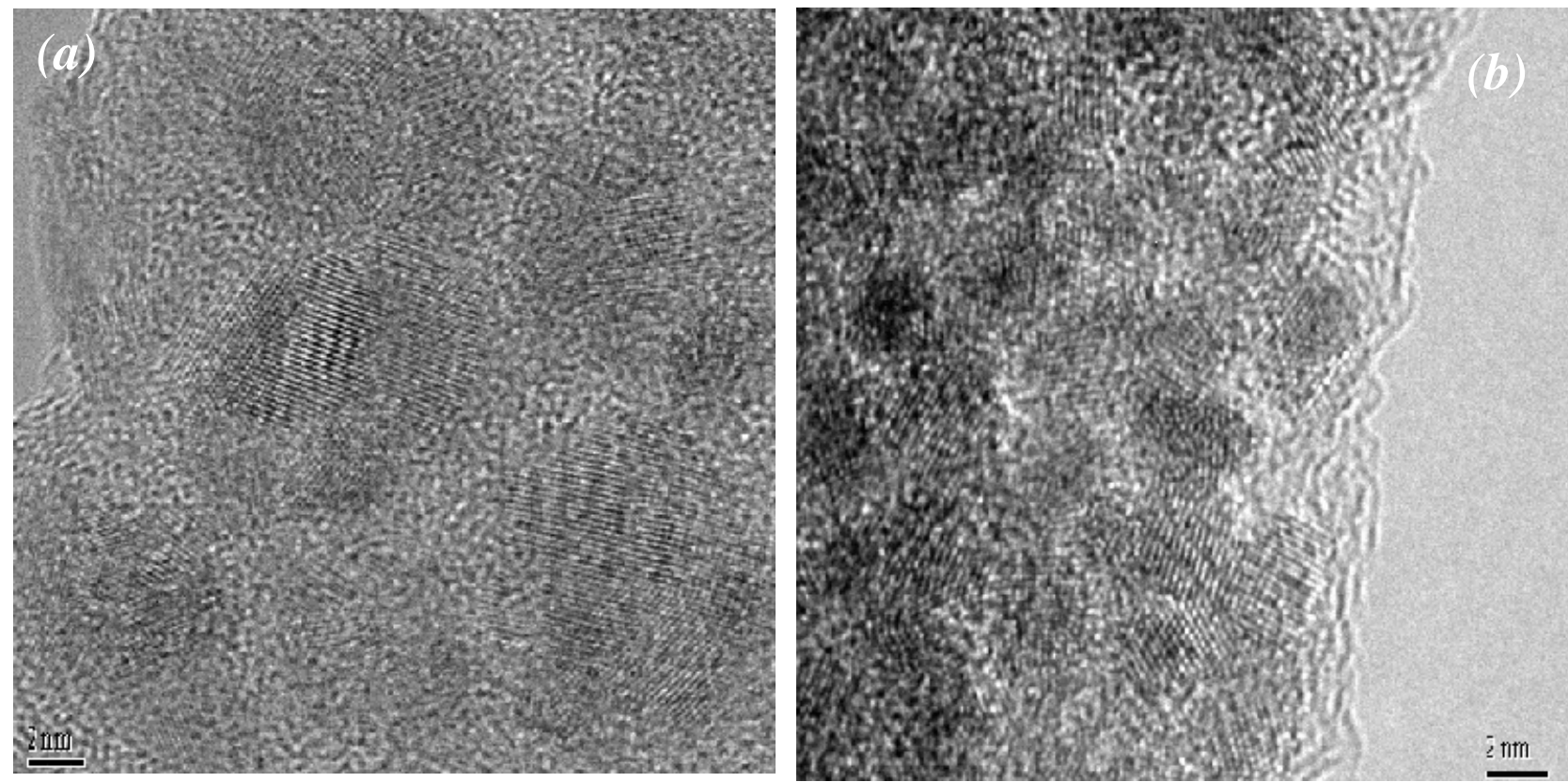

Figure 1. HREM images of pure (a) and Ru-doped (b) DLC thin films.

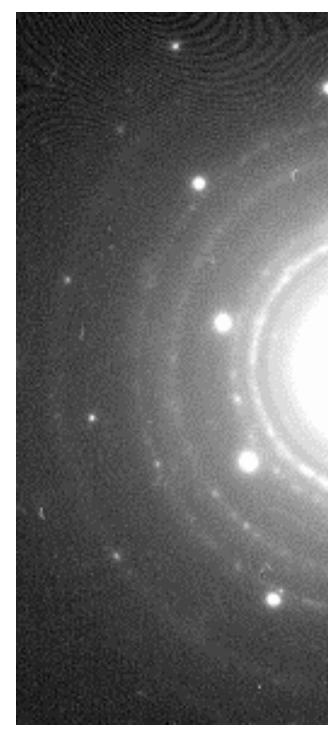

(a)

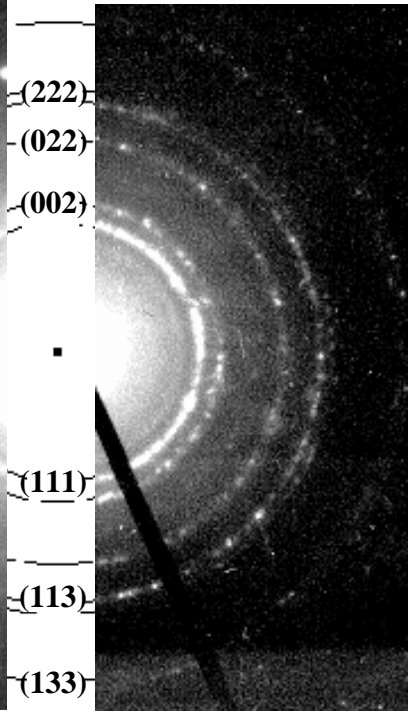

(c)

Figure 2. Selected area electron diffraction (SAD) pattern of (a)pure and (c) Ru-doped DLC thin films, and (b)n-diamond indexing

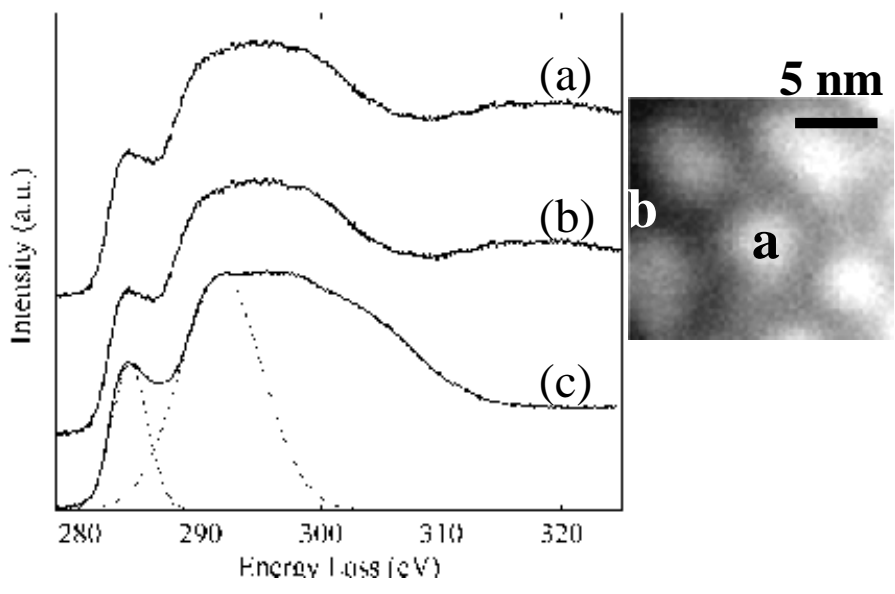

Figure 3 The inset is a portion of the Zcontrast image. The carbon K edge collected from (a) NCD area and (b) matrix area of $\mathrm{Ru}$-doped thin film, and (c) C60. The $\pi^{*}$ and $\sigma^{*}$ peaks were fitted with a Gaussian function as indicated by the dashed lines. 